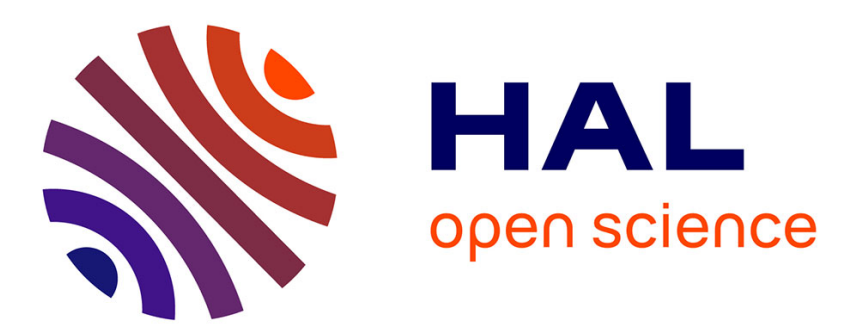

\title{
Theoretical interpretation of conductivity data below and above the CMC: The case of alkaline ion decanoate solutions
}

\author{
S. Durand-Vidal, O. Bernard, Ž. Medoš, M. Bešter-Rogač
}

\section{- To cite this version:}

S. Durand-Vidal, O. Bernard, Ž. Medoš, M. Bešter-Rogač. Theoretical interpretation of conductivity data below and above the CMC: The case of alkaline ion decanoate solutions. Journal of Molecular Liquids, 2020, 309, pp.112968. 10.1016/j.molliq.2020.112968 . hal-02859995

\section{HAL Id: hal-02859995 \\ https://hal.sorbonne-universite.fr/hal-02859995}

Submitted on 8 Jun 2020

HAL is a multi-disciplinary open access archive for the deposit and dissemination of scientific research documents, whether they are published or not. The documents may come from teaching and research institutions in France or abroad, or from public or private research centers.
L'archive ouverte pluridisciplinaire HAL, est destinée au dépôt et à la diffusion de documents scientifiques de niveau recherche, publiés ou non, émanant des établissements d'enseignement et de recherche français ou étrangers, des laboratoires publics ou privés. 


\section{Theoretical interpretation of conductivity data below and above the CMC: The case of alkaline ion decanoate solutions}

S. Durand-Vidal ${ }^{a *}$, O. Bernard ${ }^{a}, \check{Z}$. Medoš ${ }^{b}$, M. Bešter-Rogač ${ }^{b}$

${ }^{a}$ Sorbonne Université, Faculté des Sciences et d'Ingénierie - Campus Pierre et Marie Curie. Laboratoire PHENIX - UMR CNRS 8234. CC 51, 4 place Jussieu 75252, Paris cedex 05, France.

${ }^{b}$ Faculty of Chemistry and Chemical Technology, Aškerčeva 5, University of Ljubljana, Sl-1000 Ljubljana, Slovenia.

* E-mail address: serge.durand-vidal@sorbonne-universite.fr

Keywords: decanoates, electrical conductivity, association, micellization, mean spherical approximation. 


\begin{abstract}
In this work the conductivity data of three carboxylate surfactant systems: sodium (NaDec), potassium (KDec) and cesium (CsDec) decanoates aqueous solution in the concentration range $10^{-3}-0.25 \mathrm{~mol} \mathrm{dm}^{-3}$, between temperatures 278.15 and $328.15 \mathrm{~K}$, are analyzed by a transport theory in the frame of the mean spherical approximation (MSA). The data in the pre-micellization region, below critical micelle concentration $(\mathrm{CMC})$, are reproduced well by taking into account the formation of neutral pairs involving monomers and counterions. It was found, that the values of the thermodynamic association constants, $\mathrm{K}$, for $\mathrm{NaDec}$ and KDec are very similar and slightly smaller than values of $\mathrm{K}$ for $\mathrm{CsDec}$. In all cases, $\mathrm{K}$ decreases with increasing temperature. Above CMC, the fitting of experimental data yields the effective charge of micelles, which also turned out as counterion quite insensitive, but is bigger at higher temperatures. Thus, at higher temperatures below $\mathrm{CMC}$ the presence of free monomers and counterions is favorable and above CMC the binding of counterion on the micelle surface is weaker. However, at studied systems no distinctive ion specific effect could be observed, thus the dominant role Dec- as a surfactant monomer in the micellization process could be assumed.
\end{abstract}

\title{
1 Introduction
}

Electrical conductivity experiments were performed previously to study three micellar systems from 278.15 to $328.15 \mathrm{~K}$ with step of $10 \mathrm{~K}$ : Sodium, Potassium and Cesium Decanoates (NaDec, KDec and CsDec) below and above their Critical Micellar Concentration (CMC) [1]. Electrical conductivity measurements of surfactant solutions are commonly used to determine the value of their critical micelle concentration (CMC). Below the CMC, it is generally assumed that the counterions and monomers of amphiphilic molecules are present as free charged species in solution. Thus, if the solution is sufficiently diluted, they should behave as "simple electrolytes" and the experimental conductivity data can be treated by help of existing models, in order to deduce from these measurements the individual limiting equivalent conductivities of the ions in solutions. However, in order 
to obtain a good precision it is necessary that the measurements were carried out in very dilute solutions ( $<10^{-2} \mathrm{~mol} \mathrm{dm}{ }^{-3}$ for 1-1 salts). Previous experiments [1] were conducted at concentrations too high to allow for sufficient accuracy. On the other hand, there is little conductivity data, in the literature, for these solutions at low concentrations and for all the temperatures considered. Measurements were made a long time ago at low concentrations at $298 \mathrm{~K}[2]$. More recently, new experiments have been carried out at low concentrations for some of the temperatures studied [3]. Then in this work, additional experiments were carried on diluted solutions of $\mathrm{NaDec}$, to improve the reliability of the study. Moreover, to closely analyze all these experimental data, we have used a theoretical description of conductivity previously developed to describe electrolytes and surfactants in solution $[4,5]$. This approach has allowed determining the aforementioned quantities characteristic of ions at low concentrations, as well as those related to micellization process.

Beyond the CMC, micelles are formed by the combination of $n_{\text {agg }}$ (aggregation number) monomers and possibly several counterions, bound on the surface. Because the micelles have usual lower conductivity than the monomers (and counterions) that constitute them, the conductivity of its solution is decreasing. In the case, that the amphiphilic ions contain a long hydrocarbon chain, the CMC is very low and thus below (and slightly above) the $\mathrm{CMC}$, the solution is very diluted. For diluted ionic surfactant solutions, it is usually assumed that their specific conductivities are linear dependent on the concentration. After the $\mathrm{CMC}$ the proportions of the various ions (micelles, monomers, counterions) vary and thus the slope of the specific conductivity as a function of the concentration is different. From the intersection of two straight lines on the conductivity vs. concentration plots above and below the change in the slope the CMC can be determined [6].

However, the quantitative description of the conductivity of ionic surfactant solutions, as well as that of electrolyte solutions, is not such an easy task. For a binary electrolyte, the fact that its specific conductivity is assumed to vary linearly with its concentration implies that its molar conductivity should be constant with the concentration. From a theoretical point of view, this behavior is expected only for ideal solutions (without interactions between ions in solution). Indeed, since Debye and Onsager, it is well known that 
the molar conductivity of diluted solutions decreases with the concentration according to a law starting with a term proportional to the square root of the concentration [7]. At higher concentrations the interactions between charged species in solution tend to decrease their ionic mobilities. As a result, the specific conductivity does not vary linearly with the concentration any more. In order to interpret this behavior more quantitatively, a model deduced from transport theories for electrolyte solutions was developed to describe the conductivity of surfactants, where the charged species are regarded as charged hard spheres for which Coulomb interactions and individual excluded volumes are considered. This model has proved to be suitable to account for the evolution of the conductivity of solution like sodium dodecyl sulfate (SDS) or dodecyltrimethylammonium bromide (DTABr) [8], where no association below CMC was taken into account. When the surfactant molecule has a shorter alkyl chain, the CMC becomes higher and the solutions even below (at least near) the CMC can no longer be considered as ideal. Thus, as in concentrated electrolyte solutions, deviations from ideality must be taken into account to quantitatively describe the changes of conductivity with concentration.

For surfactant solutions it is assumed, that small aggregates of monomers and counterions are formed even before CMC (premicellization processes), acting as precursors for micelles. This behavior can only be observed for systems with relatively high CMC, therefore with ionic surfactants having shorter alkyl chain, as for example with decyl or dodecyl ammonium chlorides [5]. In that work, various mechanisms were considered to explain premicellization, among them the formation of a neutral pair followed by an association involving two monomers and a counterion turned out to be the most probable first step in the premicellization process. For tetradecyl ammonium chloride with CMC below $0.01 \mathrm{M}$ it turned out, that the association process below CMC could be neglected [5]. Otherwise, at concentrations far above the CMC, various changes of charges, sizes and conformation of micelles can appear. These changes, observable in more concentrated solutions, have not been considered in this study.

In present work, we extended our investigations to NaDec, KDec and CsDec aqueous solutions as model systems for cationic surfactants. Electrical conductivity measurements have been performed, previously [1] and in this study, from 278.15 to $328.15 \mathrm{~K}$ with 
step of $10 \mathrm{~K}$ below and above their CMC. In order to interpret the conductivity data, the interactions between charged species is described in the frame of the mean spherical approximation (MSA) with association. The transport model derived from this treatment of the interactions, provides (besides other parameters) the association constants below the CMC and effective charges of micelles above the CMC. In addition, the variation of dissociation degrees of cations according to their nature and the temperature will be discussed.

The paper is organized as follows. The experimental procedure is described in the next section. Then in section 3 the theoretical treatment of the electrical conductivity is presented. Finally, the main results and highlighting advantages and limitations of our approach and improvements will be presented and discussed.

\section{Experimental procedure}

In order to estimate the diffusion coefficients of decanaote anion, additional measurements of electrical conductivity below CMC for NaDec water solutions were performed. Solutions were prepared form pure solid NaDec, obtained by the synthesis described in our previous work [1] and were transferred in the set of cells with volumes from 8 to $30 \mathrm{~cm}^{3}$ with the cell constants from 3.3 to $85 \mathrm{~cm}^{-1}$.

The conductivity cells were calibrated using dilute potassium chloride solutions and immersed in a high precision thermostat . The silicon oil bath was set to each temperature with a reproducibility $<0.005 \mathrm{~K}$ and stability of $0.003 \mathrm{~K}$. The temperature was also checked using a calibrated Pt100 resistance thermometer (MPMI 1004/300 Merz) connected to an HP 3458 A multimeter. An in-house developed software package was used for temperature control and the acquisition of conductance data. The measurement regime, including corrections and extrapolation of the sample conductivity to an infinite frequency, is described in Ref [9]. After taking into account experimental error (calibration, measurements and impurities), the relative standard uncertainty of specific conductivity is about $0.5 \%$. All experiments were carried out at atmospheric pressure, p $=0.1 \mathrm{MPa}$. Molar conductivities were calculated and further used in data analysis. 
The experimental data are presented in Fig. 1 and in Table S1 in Supporting Informations. As usual, in order to determine molarities $C$ (in mol $\mathrm{dm}^{-3}$ ) and molar conductivities $\Lambda$, from molalities $m$ (in mol kg-1 of solvent) and specific conductivities $\chi$, we used density measurements of $\mathrm{NaDec}$, KDec and $\mathrm{CsDec}$, determined previously and given in the Supporting Informations of [10] (see eq. (xxxiii), tables S2 and S3). Then, knowing the molar mass $M$ of the sodium decanoate $\left(M=194.25 \mathrm{~g} \mathrm{~mol}^{-1}\right)$ and the densities $\rho$ of the solutions, the molarities $C$ were deduced from the molalities $m$ using the usual relation [11]. Given that $\rho$ is dependent on the temperature $T$, the determination of the concentration $C$ associated with $m$ must be carried out for each of the temperatures considered. Taking into account that $\rho$ is very close to water density for the dilute solutions for which the measurements were carried out in this study, it can be notice that $C \simeq m$. However, this relation remains particularly necessary to determine the molarities $C$ corresponding to the molalities $m$ of the conductivities previously measured at higher concentrations [1]. Next, the molar conductivity $\Lambda$ were deduced from $C$ and the measured $\chi$, using the relation: $\Lambda=\chi / C$. Values of $C$ and $\Lambda$ are given in supplementary data of this article: Tables S3 for NaDec, S4 for KDec and S5 for CsDec. Corresponding curves of the two last systems for each temperatures are also given: Figure S1 for KDec and Figure S2 for CsDec. Parameters used do reproduce conductivities of these systems can be find in Table S2.

\section{Description of the conductivity of ionic surfactant solutions}

The theoretical description of electrical conductivity of solution used here is based on Onsager's continuity equations [7]. It treats the solution in the framework of a primitive model, that assumes the solvent to be a continuous medium characterized by its dielectric constant and its viscosity. Moreover ions are assumed to be charged hard spheres. Ionic distribution at equilibrium is described by the mean spherical approximation (MSA) [12] and a linear perturbation technique is used to describe the dynamic properties [13]. The resulting theory is thus called the MSA-transport theory. It was successfully used to de- 
scribe conductivity of simple binary electrolytes with mean radii [14] and with individual radii [13]. It has also been extended to describe the conductivity of solutions containing three different simple ionic species [15] or more [16]. Within this approach, pair interactions between two species $i$ and $j$ include Coulomb interaction $V_{i j}^{C b}$ and Hard Sphere repulsion $V_{i j}^{H S}$ given by:

$$
V_{i j}^{C b}=\frac{e_{i} e_{j}}{4 \pi \varepsilon_{0} \varepsilon_{r} r_{i j}}
$$

and

$$
V_{i j}^{H S}=\left\{\begin{array}{cc}
\infty & \text { if } r_{i j}<\sigma_{i j} \\
0 & \text { if } r_{i j}>\sigma_{i j}
\end{array}\right.
$$

Where $e_{i}$ is the charge of ion $i, \varepsilon_{0}$ is the dielectric constant of the vacuum, $\varepsilon_{r}$ is the relative dielectric constant of water and $r_{i j}$ is the distance between species $i$ and $j$. Each species $i$ has its individual diameter $\sigma_{i}=2 r_{i}$, with $r_{i}$ its radius which correspond to its individual distance of closest approach and then $\sigma_{i j}=r_{i}+r_{j}$ is the closest distance of approach between ions $i$ and $j$. Values of $\varepsilon_{r}$ as a function of the temperatures are taken from the literature [17] and are given in Table 1.

Thus the description of the conductivity data also the individual radii $r_{i}$ of ions are needed. This MSA approach was tested for many simple ions and the same set of parameters (radii and diffusion coefficients) allowed to reproduce many equilibria and transport properties [18]. It permits also to reproduce experimental conductivity up to $1 \mathrm{~mol} \mathrm{dm}^{-3}$ for simple salts [14], was validates by comparison with Brownian Dynamics [4, 19, 20] and applied to more complex systems as inorganic nanocolloids [21], organic colloids [22], and micellar systems $[5,8]$. In the frame of this approach, the specific conductivity of the solution is given by the expression [4]:

$$
\chi=\frac{e^{2}}{k_{B} T} \sum_{i=1}^{3} n_{i} D_{i}^{o} Z_{i}^{2}\left(1+\frac{\delta k_{i}^{r e l}}{k_{i}}\right)\left(1+\frac{\delta v_{i}^{\text {hyd }}}{v_{i}^{o}}\right)
$$

Where $k_{B}$ is the Boltzmann constant, $T$ the absolute temperature, $e$ is the elementary charge, $Z_{i}$ the valency of the species $i\left(e_{i}=Z_{i} e\right), n_{i}$ its number density (in $\mathrm{m}^{-3}$ ) and $D_{i}^{o}$ its diffusion coefficient at infinite dilution. $\delta k_{i}^{r e l} / k_{i}$ is the relaxation correction that accounts for electrostatic friction and $\delta v_{i}^{h y d} / v_{i}$ is the hydrodynamic or electrophoretic correction. 
The calculation of electrical conductivity is based on the assumption that the dominant forces which determine the deviations from ideal behavior (i.e. without any interaction between ions) are the relaxation and electrophoretic forces [7]. The first appears because the ionic equilibrium distribution is perturbed by the external force (electric field) and consequently the electrostatic forces tend to restore the equilibrium distribution of ions. In addition, when external forces are applied to the solution, the different ions have different drift velocities. Hydrodynamic interactions of ions are mediated by the solvent and this effect is called the electrophoretic effect. Explicit formulae of those terms were given previously [14]. In this article we chose to present the molar conductivity $\Lambda$ as function of the square root of the total monomer molar concentration $C$. $\Lambda$ is given by $\Lambda=\chi / C$. In the conductivity formula given by Eq. (3) it can be seen that the conductivity is a function of the concentration of free charged species, their charge and their diffusion coefficient at infinite dilution.

Some input parameters such as the charge of ions, the distance of closest approach of ions and their diffusion coefficient at infinite dilution are needed to calculate the conductivity by help of Eq. (3). For simple cations $\left(\mathrm{Na}^{+}, \mathrm{K}^{+}\right.$and $\left.\mathrm{Cs}^{+}\right)$, diffusion coefficients at infinite dilution are well known in our temperature range [11] and are given in Table 1.

The conductivity data of diluted solutions of NaDec were analyzed in the frame of MSA approach, yielding the data on limiting value of molar conductivity, $\Lambda_{N a D e c}^{0}$, at all investigated temperatures. By help of literature data on limiting ionic conductivities for $\lambda_{N a^{+}}^{0}$ [11], the limiting ionic conductivities of decanaote anion $\mathrm{Dec}^{-}$were determined.

By help of the Nernst-Einstein law:

$$
D_{i}^{0}=\frac{k_{B} T}{N_{A} e^{2}} \lambda_{i}^{0}
$$

Where $N_{A}$ is the Avogadro number, the diffusion coefficients of decanoate anion, $D_{D e c^{-}}^{0}$ were determined, which is connected also to the hydrodynamic radius, $r_{D e c}^{H}$, by the StokesEinstein relation

$$
r_{D e c}^{H}=\frac{k_{B} T}{6 \pi \eta D_{D e c}^{0}}
$$

Where $\eta$ is the viscosity of water. All these values are gathered together with the viscosities and relative permittivities of water in Table 1 . The individual distances of closest approach 
have been determine previously at $298.5 \mathrm{~K}$ for simple ions $[14,16]: r_{N a^{+}}=1.1710^{-10} \mathrm{~m}$, $r_{K^{+}}=1.7010^{-10} \mathrm{~m}$ and $r_{C^{+}}=1.6710^{-10} \mathrm{~m}$, and were kept as constants at all the temperature for the three systems.

As previously stated, in order to adequately describe surfactant solutions it is necessary to consider the formation of micelles beyond the $\mathrm{CMC}$, which can be described by the chemical equilibrium

$$
n M^{-}+m C^{+} \rightleftharpoons\left(M_{n} C_{m}\right)^{m-n} \quad K_{n m}=\frac{\left(M_{n} C_{m}\right)}{\left(M^{-}\right)^{n}\left(C^{+}\right)^{m}}
$$

Where $n$ is the number of monomers $M^{-}$and $m$ of counterions $C^{+}$composing the micelle. This chemical equilibrium enables the description of any variations in concentrations of monomers, counter-ions and micelles in the vicinity of the CMC. However, in the first approximation, when the total concentration of surfactant $C$ is much lower than the $\mathrm{CMC}$, the micelle concentration is of the order of $K_{n m} C^{(n+m)}$ and is therefore negligible compared to the monomer concentration, which is of the order of $C-n K_{n m} C^{(n+m)}$. Then, when only the previous chemical equilibrium is considered, aggregates smaller than the micelles, formed before the $\mathrm{CMC}$, are not taken into account. In particular, the formation of neutral pairs during the association between a cation and an anion here is neglected while this is an usual phenomenon in solution of symmetrical electrolytes. These premicellar aggregates can have a great impact on the amplitude of the measured conductivity. Indeed, in view of the Eq. (3), the conductivity value depends largely on the number densities of small ions as they are the most mobile. In particular, the formation of neutral pairs, not participating to the current, induces a sharp decrease of this quantity.

Different premicellar species formed by smaller numbers of monomers and counterions could be also taken into account with the same types of chemical equilibria but the number of fitting parameters would not be reasonable. In our previous study on ionic surfactants having different chain lengths [5], we investigated the influence of several chemical equilibria that can represent the smallest aggregates formed at the beginning of the premicellization phenomenon. Several alternative processes were considered:

- The first proposed association process involved first the formation of a dimer consisting of two monomers and then the association of a counterion on this dimer. 
- As an alternative, a second association process first considered the formation of a neutral dimer consisting of a monomer and a counterion, and then the addition of a second monomer to that dimer.

We have shown that the first association process did not allow to account for the conductivity variations observed before the CMC, unlike the second association process considered [5]. Moreover, in order to simplify the description of the premicellization and to reduce the number of parameters to be adjusted, we finally considered the description of the premicellization by taking into account only the first equilibrium of the second process, namely the formation of neutral ion pairs between monomers and counterions. This single chemical equilibrium also allowed to account for the observed conductivity below the CMC. Now in this study, in order to describe the premicellization observed for alkanoates of alkali ions, for the same reasons, we have also taken into account only the formation of neutral pairs between monomers and counterions.

\subsection{Below the CMC}

Below the CMC or - more precisely - at lowest concentrations, the solution of any ionic surfactant is similar to that of a fully dissociated binary electrolyte and the linear dependence on the square root of concentration can be observed (Onsager limiting law) [7], as it is also clearly evident on Fig. 1.

For more concentrated solutions, where the limiting law no longer applies, theory needs to consider the sizes of the ions and eventually of other short range interactions such as association. First, an individual diameter $\sigma_{D e c^{-}}$must be assigned to the decanoate ion, in order to describe its interactions with the other ions. For simplicity, we have chosen to connect this diameter to the hydrodynamic radius: $\sigma_{D e c^{-}}=2 r_{D e c^{-}}^{H}$. In addition, to better describe the measured conductivity, we also considered an association between decanoate ions $D e c^{-}$and their counterions $C^{+}$.

$$
\left(C^{+}\right)+\left(D e c^{-}\right) \rightleftharpoons(C D e c)
$$

The concentrations of free ions, $\left[C^{+}\right]$and $\left[D e c^{-}\right]$, and of neutral pairs $[C D e c]$ were cal-

culated assessing the effect of the activity coefficients $\gamma_{j}$ of the species $j$ involved in the 
chemical equilibrium.

$$
\mathrm{K}=\frac{(C D e c)}{\left(C^{+}\right)\left(D e c^{-}\right)}=\frac{[C D e c]}{\left[C^{+}\right]\left[D e c^{-}\right]} \frac{\gamma_{C D e c}}{\gamma_{C^{+}} \gamma_{D e c^{-}}}
$$

The ionic strength and the closest distance of approach are taken into account in this description. The exact procedure and equations used to evaluate the activity coefficients were already given in a previous article [5]. Results concerning $\mathrm{K}$, the deduced association constant, are summarized for the three systems in Table 2. In addition, we note that the association constant is adjusted at each temperature. The variation of the interactions with the temperature is thus taken into account in this way.

\subsection{Above the CMC}

Above this concentration, in order to calculate the conductivity with the equation (3), three species are taken into account: the micelles, the free decanoate ions and the free counterions. The proportions of the various species are determined using the pseudo-phase model as described in the previous study [5]. The description of the solution is characterized by three quantities. Obviously, the first is the critical micellar concentration, above which a significant amount of micelle is considered. The second is the aggregation number, number of monomers associated to form a micelle $\left(n_{a g g}\right)$. The micellar concentration $C_{M i c}$ is deduced using the aggregation number $n_{a g g}$ : $C_{M i c}=\left(C-C_{D e c^{-}}\right) / n_{a g g}$, were $C$ is the total concentration of surfactant solution and $C_{D e c^{-}}$is the concentration of free decanoate ions (= CMC in the pseudo-phase model). The ionic mobility of the micelles is calculated from the value of their diffusion coefficient $D_{M i c}^{0}$ and of their effective charge $Z_{M i c}$. The effective charge is determined by considering that a part of the counterions are linked to the micelles and move like them. Then the third quantity, necessary to characterize the charged micelles, is their degree of dissociation $\alpha$. The effective charge can be deduced

from the aggregation number $n_{a g g}$ and the dissociation degree $\alpha,\left|Z_{\text {Mic }}\right|=\alpha n_{a g g}$. The dissociation degree $\alpha$ is needed also to evaluate the concentration $C_{+}$of free counterions. The concentration $C_{+}$is given by: $C_{+}=C_{D e c^{-}}+\alpha\left(C-C_{D e c^{-}}\right)$. Therefore, to quantify the micellization process, the aggregation number $n_{\text {agg }}$ has to be known. We sought to have a description of micellization as consistent as possible with results of the two-step micel- 
lization model applied to isothermal titration calorimetry (ITC) experimental data [10]. In this previous study, however, a much more detailed description of micelle formation was developed. Namely, two types of micelles were considered and the obtained average aggregation numbers from these two kinds of micelles were also temperature dependent and changed with concentration. From the proportions and the aggregation numbers of the two micelles, an average aggregation number was deduced for each temperature. On the other hand, the degree of dissociation $\alpha$ was independent of the temperature. The direct application of this model would have led us to consider a very large number of additional parameters. In order to reduce the number of parameters necessary to characterize the micellization in our study, we chose to consider only one type of micelles with a single number of aggregation independent of the temperature. The value retained for the three systems $\left(n_{a g g}=12\right)$, roughly corresponds to an average value of the average aggregation numbers determined in the previous study at each temperature. On the other hand, we have taken possibly different degrees of dissociation, at each temperature, for the three systems, in order to best account for the experimental variations of conductivity. In addition, to characterize the micelles we need to assign them a diffusion coefficient and an individual diameter $\sigma_{M i c}$ in order to calculate the relaxation and hydrodynamic corrections. For simplicity, both were deduced from a size determined from the chemical structure of the decanoate ions. The size of micelles was estimated by Eq. (3) in reference $[23]:$

$$
r_{M i c}=1.50+1.26 n_{C} \quad(\text { in } \AA)
$$

Where $n_{C}$ is the carbon number in the alkane chain, equal to nine in the present case. We found a value of $1.2810^{-9} \mathrm{~m}$ for $r_{M i c}$. The radius $r_{M i c}$ is supposed to be the hydrodynamic radius $r_{M i c}^{H}$ of the micelles. We used the Stokes-Einstein relation to deduce $D_{M i c}^{0}$ from $r_{M i c}^{H}$, taking into account the temperature dependence of the viscosity as given by data in [24]. The individual diameter is also deduced from $r_{M i c}$ with the relation $\sigma_{M i c}=2 r_{M i c}$. All parameters used to reproduce experimental results are summarized for the three systems in Tables 1, 2 and Table S2 in Supporting Informations. 


\section{Results and discussion}

The experimental data determined in this study are presented in the Fig. 1 together with literature experimental values determined previously $[2,3]$. There is a very good agreement between our measurements and those made by Kinart [3]. On the other hand, the data previously determined by Campbell [2] differ more from the values obtained in this work or in [3]. For comparison, we have also given in Table 1 the values of limiting conductivities of decanoate determined previously [3], at the same temperatures as in this study. Here again, we observe a very good agreement with our values. In Fig. 2 the experimental data in the whole concentration range (below and above CMC) for NaDec water solution at $298.15 \mathrm{~K}$ are presented together with the results obtained by different fitting procedures: using MSA-transport calculations without association $(\mathrm{K}=0)$ and with association $(\mathrm{K}=0.7)$ below the $\mathrm{CMC}$, and with different micellar charge $(-7,-8$ and -9) above the CMC. The parameters, needed to obtain the theoretical curves are CMC = 0.110 mol. $\mathrm{dm}^{-3}$, corresponding diffusion coefficients $\left(D_{\mathrm{Na}^{+}}^{\circ}, D_{\mathrm{Dec}^{-}}^{\circ}, D_{\mathrm{Mic}}^{\circ}\right)$ and individual radii $\left(r_{\mathrm{Na}^{+}}, r_{\mathrm{Dec}^{-}}, r_{\mathrm{Mic}}\right)$ which are taken from Table 1.

The comparison of experimental and fitted data in whole investigated temperature range is presented in Fig. 3 for NaDec, and Figs. S1 and S2 for KDec and CsDec, in the Supporting Informations part. The comparison for all three investigated systems at 298.15 K is given in Fig. 4. In Table 2 all obtained parameters are listed, together with the literature data on CMC as obtained from conductivity [1, 2], ITC experiment [10] and other studies [25]. Some of these concentrations were determined previously in moles of surfactant per $1000 \mathrm{~g}$ of water (molality). In order to compare with the values given in this study we used again the density measurements of NaDec, KDec and CsDec solutions found in [10] to deduce the corresponding values of $\mathrm{CMC}$ in $\mathrm{mol} \mathrm{dm}^{-3}$. The greatest differences are observed at the lowest temperatures. As it can be seen in Fig. 3, the slope difference, before and after the $\mathrm{CMC}$, is very small at low temperatures. In consequence, the determination of the $\mathrm{CMC}$ is less precise in these conditions, especially when one represents the specific conductivity $\chi$ as a function of the concentration.

In this work, the literature values of CMC were used first to deduce the effective 
charge of micelles $Z_{M i c}$ and then the degree of micelle ionization or dissociation degree $\alpha$ which is equal to $\left|Z_{M i c}\right| / n_{\text {agg }}$ according to the procedure described in our previous study [5]. As it can be seen on Fig. 2, the "theoretical" slope before CMC is strongly dependent on the consideration of a possible pre-micellization association, whereas above CMC the most influential parameter is the effective charge of micelles. Thus, by the fitting procedure the association constant $\mathrm{K}$ below the $\mathrm{CMC}$ and the effective charge above the $\mathrm{CMC}$ were estimated to obtain the best agreement between experimental and calculated values of molar conductivity. Then CMC were re-evaluated from the interception and the whole process has been started again. All CMC values, given in Table 2, are in reasonable agreement despite these different approaches. Concerning the effective charge and therefore the degree of ionization $\alpha$ we can make a comparison with previous works. Values of $\alpha$ obtained in this work are distinctly bigger than those reported from ITC experiment [10] but the approach is very different. In [10] $\alpha$ was taken as temperature independent, the aggregation number change with temperature and it is a two-steps micellization model. The values that we obtain are also a little larger than those obtained by conductivity in [1] and which are of the order of 0.5-0.7. This is due to the difference in data processing. In [1] it is a comparison of the slopes before and after the CMC of the specific conductivity as a function of the concentration assuming ideal solutions, i.e. without interaction between the ions. If this approach can be used for systems with low $\mathrm{CMC}$, it leads to large deviations when CMC are important as here. However, the same trend is observed as a function of temperature. The degree of ionisation $\alpha$ increases as the temperature increases for all three systems. This seems to indicate that the association of counterions within the micelles is more important at low temperatures. This evolution can be explained qualitatively by considering that the association of counterions would depend on $\exp \left(-W / k_{B} T\right)$, where $W$ is the total interaction energy between the counterions and the charged heads of the monomers grouped in each micelle. If $W$ depends little on the temperature, the association should decrease with the temperature and the degree of ionization increase. 


\section{Conclusions}

By using a transport theory in the frame of MSA approach the conductivity data of sodium, potassium and cesium decanoates aqueous solution are analyzed in the concen-

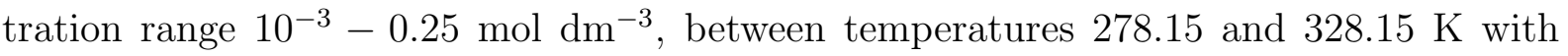
step of $10 \mathrm{~K}$. In order to estimate the diffusion coefficient of $\mathrm{Dec}^{-}$the conductivity of diluted solutions of NaDec $\left(10^{-3}-210^{-2} \mathrm{~mol} \mathrm{dm}{ }^{-3}\right)$ in the same temperature range were determined and analyzed.

The studied concentration range cover the pre- and postmicellar region of investigated systems, thus, the conductivity is not simply proportional to the concentration of charged species near and above the CMC. Moreover, due to shorter alkyl chain (C10), the CMC is higher in comparison to those usually studied for solutions of SDS or DTABr (with C12), and the premicellization phenomena (association) have to be taken into account. Finally, for those chemical systems, micelles are smaller [10] and the transition from monomers to micelles is more progressive. Therefore, the determination the CMC from the graphical representation of the specific conductivity as a function of the concentration is difficult and less reliable.

Thus, in present work a theoretical description of the molar conductivity below and above CMC was applied. In the premicellization region, the ion association, by introduction of the appropriate association constant, K, was taken into account to obtain the best agreement between experimental calculated conductivities. It was found that $\mathrm{K}$ is small for all studied systems, but very similar for NaDec and KDec and slightly higher for CsDec. Considering the Hofmeister series, it is surprising. If the carboxylate heads of the monomers are more attracted by the small dehydrated cations, it may seem indeed surprising that the cations of larger crystallographic radius (cesium) associate more than those of smaller radii. This may be due to the fact that cesium ions could dehydrate more easily than the smaller alkaline ions. However, the size chosen for cesium in our model is roughly equal to that of potassium. The association constants, determined for these two salts, should then be comparable. This indicates that the size parameter alone is insufficient to account for the specificity of the ions. In addition, the association constants have 
been adjusted for each temperature. On the other hand, the individual diameters of the counterions used in this study were taken independent of the temperature. The variation of association constants can simply reflect the evolution of interactions with temperature. If a variation in diameters with temperature had been considered, other values of the association constants would have been obtained. Finally, the fact that the association was found to be greater for $\mathrm{Cs}^{+}$than for $\mathrm{K}^{+}$and $\mathrm{Na}^{+}$, may be a consequence of the theoretical treatment used. In all cases, $\mathrm{K}$ is decreasing with increasing temperature, revealing that at higher temperatures the formation of ion pairs is less favorable.

Also estimated CMC values are very similar for all studied systems and decreasing only weakly with increasing temperature, what is in good agreement with the reported findings form ITC experiment [10]. Also, the values of the dissociation degree, $\alpha$, are very similar for all systems, but they seem to increase with the increasing temperature.

On the whole, applied transport theory in the frame of the MSA approach describes well the experimental conductivity data in the whole studied concentation range at all temperatures, but not explicit ion specific effect can be observed.

There is only some difference in CMC at low temperatures between CsDec and other two systems, whereas $\mathrm{K}$ at CsDec is systematically higher at all temperatures. However, any reliable conclusion in the direction of specific ion here is not possible. Obviously, the properties of decanoate anion with the hydrophobic alkyl chain and strongly hydrated headgroups here are prevailing and thus the nature of the counterion on the surfactant properties of these systems is less significant.

Following these results, certain perspectives can be envisaged. Same studies could be

performed for other head groups and counterions with more specific effect. Finally, it would be very interesting to be able to follow the evolution of the aggregation number, the size and the charge of these complex systems according to the temperature, the total concentration and ionic parameters.

\section{Acknowledgements:}

The four authors thank the PHC PROTEUS 2018 (Code: 39668QL and BI-FR/1819-PROTEUS -006) for the financial assistance granted to facilitate exchanges between 
the two groups. The work was partially supported by the Slovenian Research Agency through Grant No. P1-0201. 
Table 1: Temperature dependence of the values of viscosity, $\eta[24]$ and relative dielectric constant, $\epsilon_{r}$ [17] of water; diffusion coefficients at infinite dilution of ions, $\mathrm{D}_{\mathrm{Na}^{+}}^{0}, \mathrm{D}_{K^{+}}^{0}$ and $\mathrm{D}_{C s^{+}}^{0}$ deduced with Eq. (4) from the corresponding values of the limiting conductivity $\lambda^{0}$ given in [11]; ionic limiting conductivity of $\mathrm{Dec}^{-}, \lambda_{D e c^{-}}^{0}$, (as estimated from data on Fig. 1); diffusion coefficients at infinite dilution of $\mathrm{Dec}^{-}, \mathrm{D}_{\mathrm{Dec}^{-}}^{0}$, (calculated by using Eq. (4); diffusion coefficients at infinite dilution of micelles, $\mathrm{D}_{M i c}^{0}$, (obtained from Eq. (5) using an hydrodynamic radius of micelles, $\mathrm{r}_{\text {Mic }}^{H}=1.2810^{-9} \mathrm{~m}$ ).

\begin{tabular}{|c|c|c|c|c|c|c|}
\hline$T(\mathrm{~K})$ & 278.15 & 288.15 & 298.15 & 308.15 & 318.15 & 328.15 \\
\hline$\eta\left(10^{-3} \mathrm{~Pa} . \mathrm{s}\right)$ & $1.5192^{a}$ & $1.1382^{a}$ & $0.8903^{a}$ & $0.7195^{a}$ & $0.5963^{a}$ & $0.5042^{a}$ \\
\hline$\epsilon_{r}$ & $85.897^{b}$ & $82.039^{b}$ & $78.358^{b}$ & $74.846^{b}$ & $71.496^{b}$ & $68.299^{b}$ \\
\hline $\mathrm{D}_{N a^{+}}^{0}\left(10^{-9} \mathrm{~m}^{2} \cdot \mathrm{s}^{-1}\right)$ & 0.753 & 1.02 & 1.33 & 1.69 & 2.09 & 2.55 \\
\hline $\mathrm{D}_{K^{+}}^{0}\left(10^{-9} \mathrm{~m}^{2} \cdot \mathrm{s}^{-1}\right)$ & 1.16 & 1.53 & 1.96 & 2.43 & 2.94 & 3.50 \\
\hline $\mathrm{D}_{C s^{+}}^{0}\left(10^{-9} \mathrm{~m}^{2} \cdot \mathrm{s}^{-1}\right)$ & 1.24 & 1.63 & 2.06 & 2.53 & 3.05 & 3.62 \\
\hline $\mathrm{D}_{M i c}^{0}\left(10^{-9} \mathrm{~m}^{2} \cdot \mathrm{s}^{-1}\right)$ & 0.105 & 0.145 & 0.191 & 0.245 & 0.305 & 0.372 \\
\hline $\mathrm{D}_{\text {Dec }}^{0}\left(10^{-9} \mathrm{~m}^{2} . \mathrm{s}^{-1}\right)$ & 0.30 & 0.43 & 0.59 & 0.77 & 0.99 & 1.19 \\
\hline $\mathrm{r}_{\text {Dec }}^{H}\left(10^{-10} \mathrm{~m}\right)$ & 4.47 & 4.31 & 4.15 & 4.07 & 4.02 & 4.00 \\
\hline$\lambda_{\text {Dec }}^{0}\left(10^{-4} \mathrm{~S} . \mathrm{mol}^{-1} \cdot \mathrm{m}^{2}\right)$ & $12.1^{c}$ & $16.7^{c}$ & $22.2^{c}$ & $28.0^{c}$ & $34.2^{c}$ & $40.6^{c}$ \\
& - & $16.65^{d}$ & $22.51^{d}$ & $28.34^{d}$ & - & - \\
\hline
\end{tabular}

${ }^{a}$ From [24].

${ }^{b}$ From [17].

${ }^{c}$ This work.

${ }^{d}$ Data from [3] given only for comparison with our values given above. 
Table 2: Values of the fitted thermodynamic constants K, CMC, apparent charge of the micelle $Z_{M i c}$ and dissociation degree $\alpha$ as a function of temperature for sodium decanoate, with aggregation number of 12 , and comparison with literature data when available.

\begin{tabular}{|c|c|c|c|c|c|c|}
\hline$T(\mathrm{~K})$ & 278.15 & 288.15 & 298.15 & 308.15 & 318.15 & 328.15 \\
\hline $\mathrm{K}\left(\mathrm{dm}^{3} \cdot \mathrm{mol}^{-1}\right)$ & 1.0 & 0.5 & 0.4 & 0.2 & 0.1 & 0.0 \\
\hline $\mathrm{CMC}\left({\left.\mathrm{mol} . \mathrm{dm}^{-3}\right)}\right.$ & $0.135^{a}$ & $0.115^{a}$ & $0.110^{a}$ & $0.100^{a}$ & $0.100^{a}$ & $0.100^{a}$ \\
& $0.116^{b}$ & $0.107^{b}$ & $0.100^{b}$ & $0.097^{b}$ & $0.096^{b}$ & $0.098^{b}$ \\
& - & - & $0.094^{c}$ & $0.098^{c}$ & - & - \\
& - & $0.124^{d}$ & $0.113^{d}$ & $0.106^{d}$ & $0.107^{d}$ & $0.108^{d}$ \\
& - & - & $0.107^{e}$ & - & - & - \\
\hline$Z_{\text {Mic }}$ & -7 & -8 & -8 & -8 & -9 & -9 \\
\hline$\alpha=\left|Z_{\text {Mic }}\right| / n_{\text {agg }}$ & 0.58 & 0.67 & 0.67 & 0.67 & 0.75 & 0.75 \\
& - & - & $0.68^{e}$ & - & - & - \\
\hline
\end{tabular}

${ }^{a}$ This work.

${ }^{b}$ From [1].

${ }^{c}$ From [2].

${ }^{d}$ From [10].

${ }^{e}$ From [25]. 


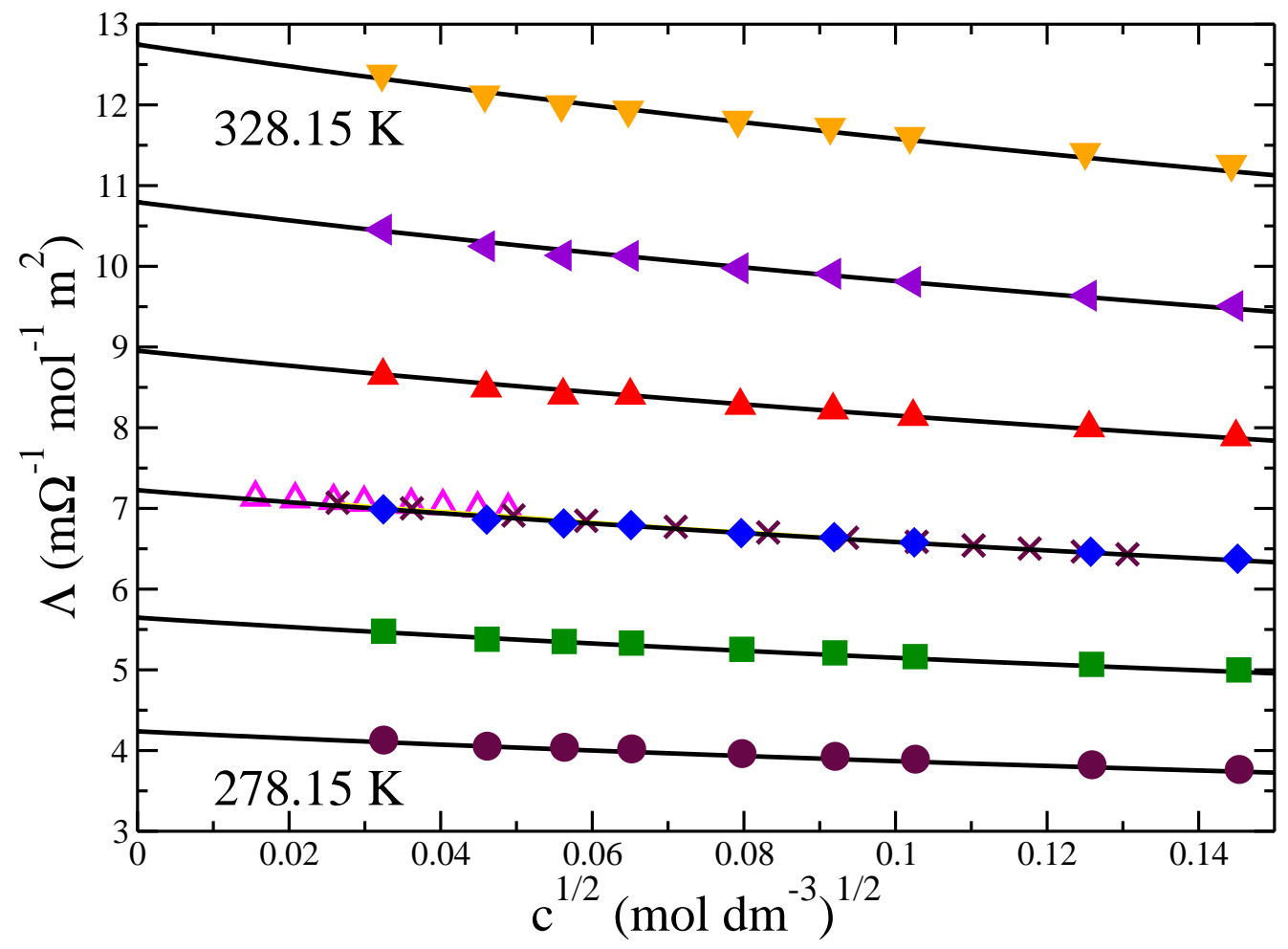

Figure 1: Molar conductivities, $\Lambda$, of NaDec solutions in water from $278.15 \mathrm{~K}$ to 328.15 $\mathrm{K}$ in steps of $10 \mathrm{~K}$ in the concentration range $0.0001<\mathrm{c}\left(\mathrm{mol} \mathrm{dm}^{-3}\right)<0.025$. Pink triangles: data from [2]; Crosses: data from [3]; Full symbols data from this work; Lines show the results of the MSA-transport fit, which give the conductivity at infinite dilution of NaDec. 


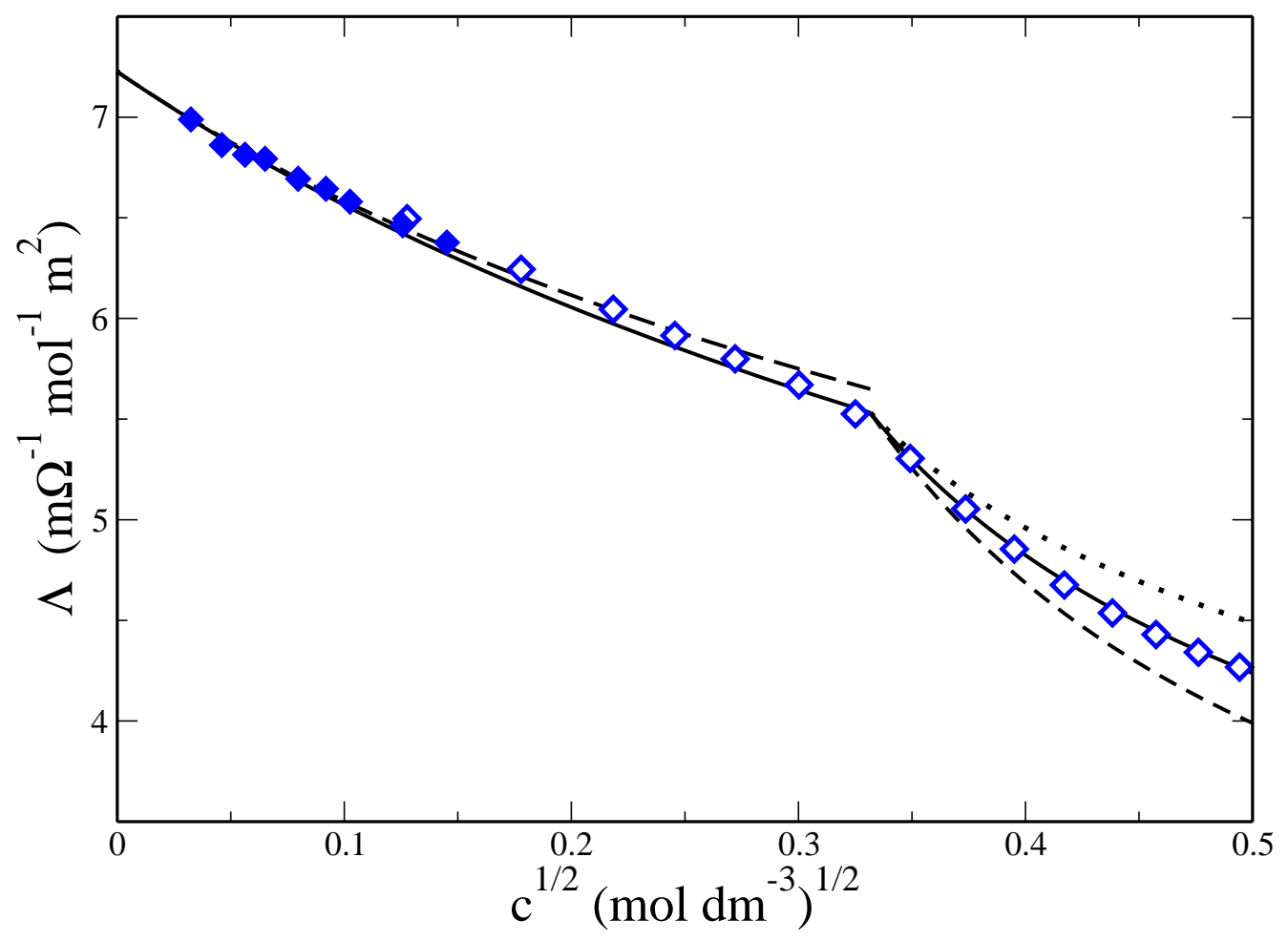

Figure 2: Molar conductivities, $\Lambda$, of NaDec solutions at $298.15 \mathrm{~K}$ as a function of the square root of the total monomer concentration c, below and above the CMC. Symbols represent experimental values and the curves are the result of our theoretical MSAtransport calculations. Full diamonds: this work; Empty diamonds : from [1]; Dashed line below the CMC: calculation without association $(\mathrm{K}=0)$; Full line below the CMC: with association $\left(\mathrm{K}=0.4 \mathrm{~L} \cdot \mathrm{mol}^{-1}\right)$; Dotted line above the $\mathrm{CMC}$ : calculation with a micellar charge $Z_{M i c}=-7$; Solid line above the CMC: with $Z_{M i c}=-8$; Dashed line above the CMC : with $Z_{M i c}=-9$. 


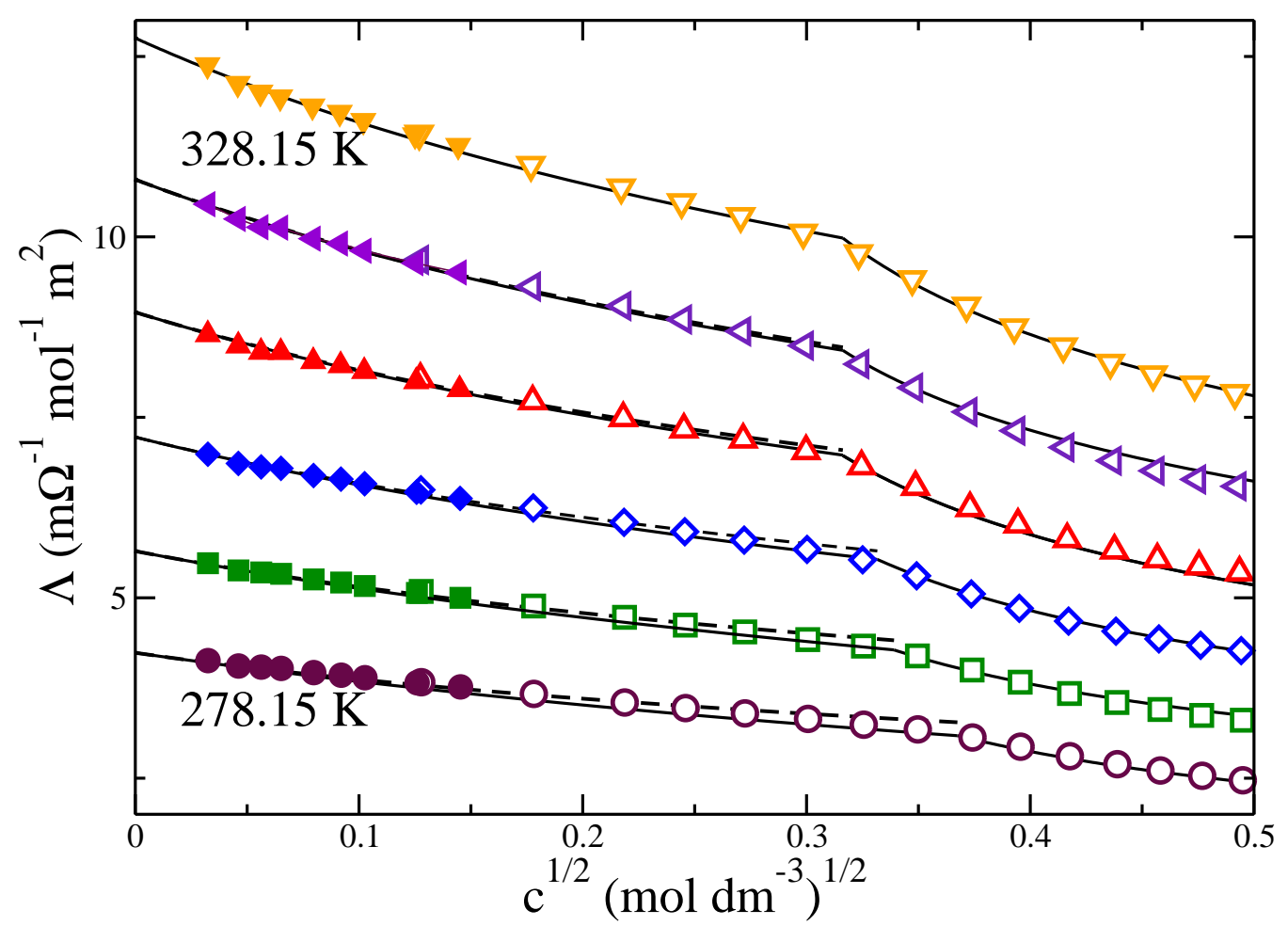

Figure 3: Molar conductivities, $\Lambda$, of NaDec aqueous solutions from 278.25 K to $328.15 \mathrm{~K}$ (in step of $10 \mathrm{~K}$ ) as a function of the square root of the total monomer concentration. The symbols represent experimental values and the curves the result of our theoretical MSAtransport calculations. Full symbols: this work; Empty symbols from [1]; Dashed lines below the CMC: calculations without association; Solid line: calculations with parameters indicated in Table 2. 


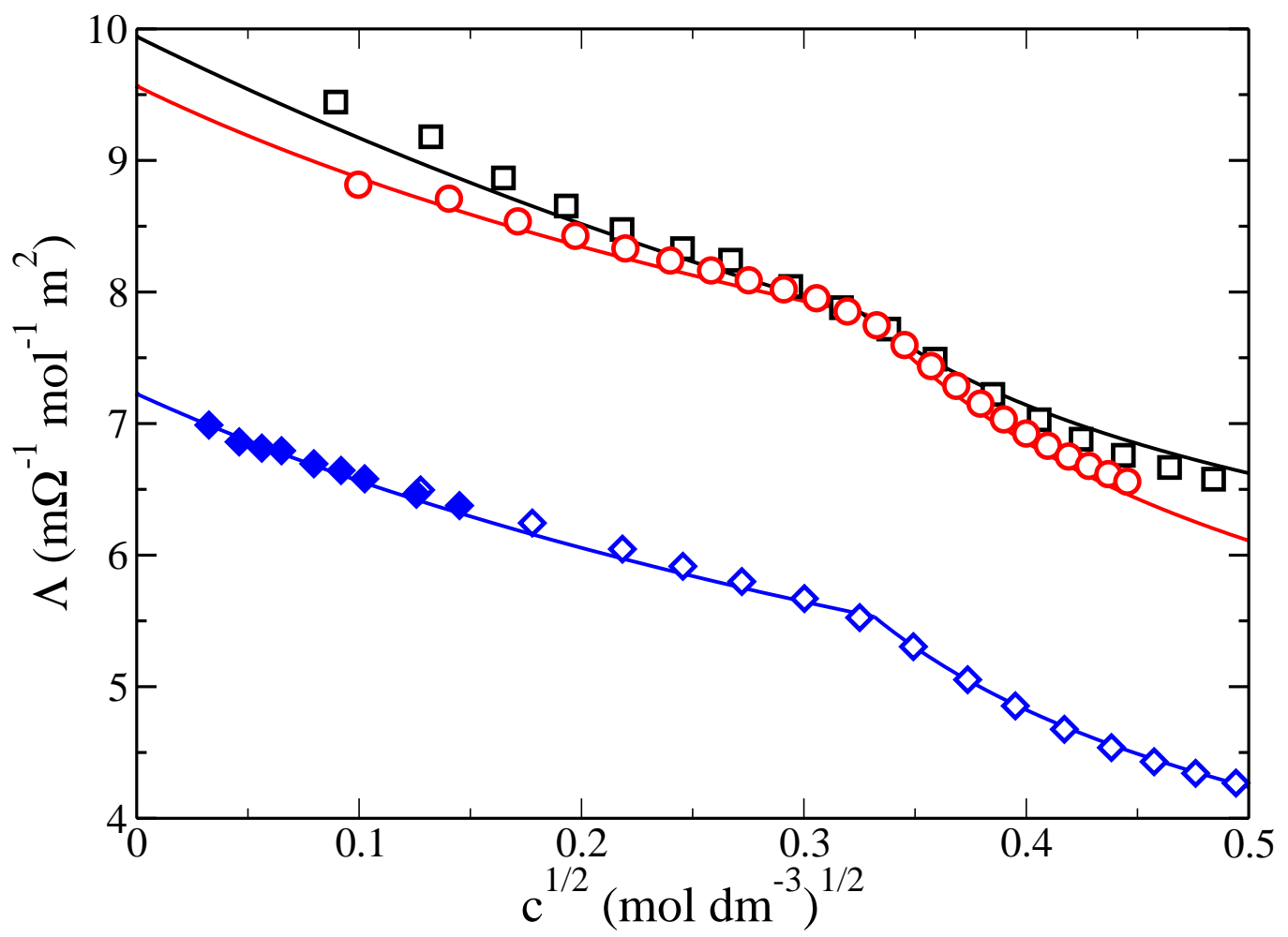

Figure 4: Molar conductivity of investigated micellar systems in water at $298.15 \mathrm{~K}$, as a function of the square root of the total monomer concentration. The lines represent the result of our theoretical MSA-transport calculations and the symbols represent experimental values. The full diamonds come from this work and the empty symbols from [1]. Upper curves: CsDec solutions (black squares). Second curve: KDec solutions (red circles). Lower curves: NaDec solutions (blue diamonds). 


\section{References}

[1] Ž. Medoš, M. Bešter-Rogač, Thermodynamics of the micellization process of carboxylates: a conductivity study, J. Chem. Thermodyn. 83 (2015) 117-122.

[2] A. N. Campbell, G. R. Lakhshminarayanan, Conductances and surface tensions of aqueous solutions of sodium decanoate, sodium laurate, and sodium myristate, at $25^{0}$ and $35^{0}$, Can. J. Chem. 43 (1965) 1729-1737.

[3] Z. Kinart, Conductance studies of sodium salts of some aliphatic carboxylic acids in water at different temperatures, J. Mol. Liq. 248 (2017) 1059-1064.

[4] S. Durand-Vidal, M. Jardat,V. Dahirel, O.Bernard, K. Perrigaud, P. Turq, Determining the radius and the apparent charge of a micelle from electrical conductivity measurements by using a transport theory: Explicit equations for practical use, J. Phys. Chem. 110 (2006) 15542-15547.

[5] G. M. Roger, S. Durand-Vidal, O. Bernard, T. M. Perger, M. Bešter-Rogač, Interpretation of conductivity results from 5 to $45{ }^{0} \mathrm{C}$ on three micellar systems below and above the CMC, J. Phys. Chem. B 112 (2008) 16529-16538.

[6] T. M. Perger, M. Bešter-Rogač, Thermodynamics of micelle formation of alkyltrimethylammonium chlorides from high performance electric conductivity measurements, J. Colloid Interf. Sci. 313 (2007) 288-295.

[7] L. Onsager, R. M. Fuoss, Irreversible processes in electrolytes. Diffusion, conductance, and viscous flow in arbitrary mixtures of strong electrolytes, J. Phys. Chem. 36 (1932) 2689-2778.

[8] S. Durand-Vidal, P. Turq, O. Bernard, C. Treiner, Model for the conductivity of ionic mixtures in the mean spherical approximation. 2. Surfactant solutions, J. Phys. Chem. B 101 (1997) 1713-1717.

[9] M. Bešter-Rogač, D. Habe, Modern advances in electrical conductivity measurements of solutions, Acta Chimica Slov. 53 (2006) 391-395. 
[10] Ž Medoš, M. Bešter-Rogač, Two-step micellization model: The case of long-chain carboxylates in water, Langmuir 33 (2017) 7722-7731.

[11] R. A. Robinson, R. H. Stokes, Electrolyte solutions, Butterworths 1965, Second edition.

[12] L. Blum, J. Høye, Mean spherical model for asymmetric electrolytes. 2. Thermodynamic properties and the pair correlation function, J. Phys. Chem. 81 (1977) 13111316.

[13] O. Bernard, W. Kunz, P. Turq, L. Blum, Conductance in electrolyte solutions using the mean spherical approximation, J. Phys. Chem. 96 (1992) 3833-3840.

[14] S. Durand-Vidal, J.-P. Simonin, P. Turq, O. Bernard, Acoustophoresis revisited. 1. Electrolyte solutions, J. Phys. Chem. 99 (1995) 6733-1738.

[15] S. Durand-Vidal, P. Turq, O. Bernard, Model for the conductivity of ionic mixtures within the mean spherical approximation. 1. Three simple ionic species, J. Phys. Chem. 100 (1996) 17345-17350.

[16] G. M. Roger, S. Durand-Vidal, O. Bernard, P. Turq, Electrical conductivity of mixed electrolytes: Modeling within the mean spherical approximation, J. Phys. Chem. B 113 (2009) 8670-8674.

[17] B. B. Owen, R. C. Miller, C. E. Miller, H. L. Cogan, The dielectric constant of water as a function of temperature and pressure, J. Phys. Chem. 65 (1961) 2065-2070.

[18] J.-F. Dufrêche, O. Bernard, S. Durand-Vidal, P. Turq, Analytical theories of transport in concentrated electrolyte solutions from the MSA, J. Phys. Chem. 109 (2005) 9873-9884.

[19] M. Jardat, S. Durand-Vidal, P. Turq, G. R. Kneller, Brownian dynamics simulations of electrolyte mixtures: Computation of transport coefficients and comparison with an analytical transport theory, J. Mol. Liq. 85 (2000) 45-55. 
[20] M. Jardat, S. Durand-Vidal, N. Da Mota, P. Turq, Transport coefficients of aqueous dodecyltrimethylammonium bromide solutions: Comparison between experiments, analytical calculations and numerical simulations, J. Chem. Phys. 120 (2004) 62686273.

[21] S. Durand-Vidal, P. Turq, L. Marang, C. Pagnoux, J. B. Rosenholm, Determination of the effective charge of different nanocolloids at high ionic strength using conductivity and acoustophoresis, Colloids Surf. A 267 (2005) 117-121.

[22] G. M. Roger, G. Mériguet, O. Bernard, S. Durand-Vidal, P. Turq, Characterization of humic substances and polyacrylic acid: A high precision conductimetry study, Colloids Surf. A 356 (2010) 51-57.

[23] J. van Stam, S. Depaemelaere, F. C. De Schryver, Micellar aggregation numbers - A fluorescence study, J. Chem. Ed. 75 (1998) 93-98.

[24] L. Korson, W. Drost-Hansen, F. J. Millero, Viscosity of water at various temperatures, J. Phys. Chem. 73 (1969) 34-39.

[25] E. Vikingstad, A. Skauge, H. Høiland, Partial molal volumes and compressibilities of the homologous series of sodium alkylcarboxylates, R6COONa-R13COONa, in aqueous solution, J. Colloid Interf. Sci. 66 (1978) 240-246. 